\title{
Pedigree Management and Assessment Framework (PMAF)
}

\author{
Kenneth A. McVearry \\ ATC-NY, \\ Cornell Business \& Technology Park, \\ 33 Thornwood Drive, Suite 500, \\ Ithaca, NY 14850 \\ kmcvearry@atcorp.com
}

\begin{abstract}
The Pedigree Management and Assessment Framework (PMAF) is a customizable framework for writing, retrieving and assessing provenance and other metadata that reflects the quality of an information object (such as a document), the relationships between information objects and resources (such as people and organizations), etc. PMAF stores metadata in a volume-efficient format using RDF (Resource Description Framework), and can write and query metadata at a fine-grained level. Once metadata has been stored in PMAF, the user can run a variety of assessments (predefined queries) to reveal particular aspects of the metadata graph. We will demonstrate the PMAF browser interface, which can be used to view the existing metadata graph for an information object; the PMAF assessment interface, which allows the user to select and run predefined queries on the metadata; and the integration of PMAF with a standard document editor and content management system.
\end{abstract}

Keywords: information pedigree, provenance, RDF, metadata graph, assessment.

\section{Introduction to PMAF}

Modern information management systems are increasingly net-centric, making more information available more quickly. In this environment, the information consumer must distinguish decision-quality information from potentially inaccurate, or even conflicting, pieces of information from multiple sources. With knowledge of the information's pedigree, or provenance, the user can establish trust in information, ensure accountability, discover sources of errors, and correct propagated errors.

The Pedigree Management and Assessment Framework (PMAF) enables the publisher of information to record standard provenance metadata about the source, manner of collection, and the chain of modification of information as it passes through processing and/or assessment. In addition, the information publisher can define and include other metadata relevant to quality assessment, such as domain-specific metadata about sensor accuracy or the organizational structure of agencies. PMAF stores this potentially enormous amount of metadata compactly and presents it to the user in an intuitive graphical format, along with PMAF-generated assessments that enable the user to quickly estimate information quality. 


\section{PMAF Background}

PMAF was initially developed through SBIR (Small Business Innovation Research) funding provided by the Air Force Research Laboratory (AFRL) in Rome, NY. The Phase I SBIR was awarded in 2005, and the Phase II was awarded in 2006 and completed in 2008. PMAF was originally created for the Joint Battlespace Infosphere (JBI) program at AFRL, which implemented an information management environment. The original scenario for application of PMAF was the creation of Air Tasking Orders including the fusion of sensor data.

Follow-on work includes the Metadata Security Assertion Framework and Evaluation (MetaSAFE) system, which was a Phase II SBIR awarded in 2008 and completed in 2010. MetaSAFE securely manages and provides access to metadata used to validate security decisions, such as whether to release a document across a security domain. MetaSAFE added pedigree assurance features to PMAF including digital signatures and hashing; hashing provides strong binding of metadata to information objects, and digital signatures provide strong attribution of metadata to the people and/or processes that generate it.

\section{Provenance Model}

The PMAF model of pedigrees includes the following concepts.

\subsection{Resource}

A resource is any nameable object that can have an associated pedigree, or can be referred to in a pedigree. People, web sites, documents, programs, etc. are all considered resources.

\subsection{Provenance Metadata}

Provenance metadata is information about how a resource was created, transformed, or used.

\subsection{Pedigree Fragment}

Provenance metadata is stored in one or more provenance repositories in the form of pedigree fragments. Each pedigree fragment contains information about a resource. A pedigree fragment consists of one or more "local" claims about the provenance of a resource. A local claim is one that involves only a single step of the creation, transformation, or use of a resource. For example, the facts that a document was created (1) by a particular person, (2) at a particular time and place, (3) by running a particular program, and (4) using particular source documents are all local facts about that document. In contrast, if document $\mathrm{A}$ was created using document $\mathrm{B}$ as a source, then the sources of document B will be considered non-local facts about A.

The complete pedigree of a document is assembled from local statements about that document, its sources, the sources of those sources, etc. Typically, the pedigree fragments for a resource will be published by the same client application that created 
or modified the resource. However, "third party" contributions to a pedigree are also possible, i.e. created by an application that is neither the writer nor the reader of the associated resource document. Allowing such third-party contributions supports the handling of objects whose sources are unknown or partially known, such as web pages. Whether the pedigree fragments are published by the creating application, or by a third-party application, provenance metadata will typically be published through the PMAF publish API.

\subsection{Root Pedigree}

A provenance repository can be queried to obtain pedigree fragments associated with a given resource. These pedigree fragments are assembled into a root pedigree for a resource. A root pedigree is a complete (relative to the information available) collection of local information associated with a resource (where local is as defined above within a single step - rather than non-remote). The complete root pedigree for a resource can be divided into two parts: source record and usage record. The source record for a resource is the local history of a creation or transformation step resulting in the current state of the resource. The usage record for a resource describes how that resource is used in the source pedigrees of other resources.

For example, if document $\mathrm{A}$ is produced using document $\mathrm{B}$ as a source, then this fact will be in the source record of A, but will be in the usage record of B. Typically, the usage records can be more difficult to assemble than the source records, because the source record only needs to be created once (when the resource is created), while the usage record can involve many different individual creation or transformation steps performed by different users at different locations and times.

\subsection{Provenance Subgraph}

A provenance subgraph is a collection of related provenance statements assembled for use by some assessment procedure. Like a root pedigree, a provenance subgraph is associated with a particular resource, but it may include information from several connected root pedigrees. For instance, a source pedigree subgraph for a resource A may include statements of the form "A has source B" as well as "B has source C". A provenance subgraph thus allows one to draw nonlocal conclusions about a resource, such as "foreign news reports contributed to this document."

There are two pure approaches to creating such provenance subgraphs: (1) create a complete pedigree, and then filter to get the desired provenance subgraph; or (2) build up the desired provenance subgraph by gathering pedigree fragments as needed. Because complete pedigrees can be huge and can be distributed among many different repositories, we consider it impractical to ever create a "complete" pedigree, so PMAF follows the latter approach. We use the word "subgraph" because these objects can be thought of as subsets of the complete pedigree, which in turn can be thought of as a potentially huge graph of relationships among resources. In PMAF we describe these relationships using RDF (discussed below).

PMAF uses a two-stage query system to assemble provenance information for use by assessments. The first stage involves querying the provenance repositories for pedigree fragments associated with a given resource; the results of this stage of the query are 
assembled into root pedigrees. The second stage of a query involves assembling appropriate root pedigrees together to make a required provenance subgraph.

\section{Provenance Represented in RDF}

In PMAF, pedigree fragments, root pedigrees and provenance subgraphs are all represented as graphs, described using the Resource Description Framework (RDF) specification. RDF supports: a) unique unambiguous identification of the entities mentioned in pedigree statements, b) exchange of machine-readable information for automated processing of pedigree, c) unlimited extensibility of terminology used to represent pedigree, and d) semantic tagging, logical inference and other automated processing of the pedigree information.

RDF represents information as a collection of statements, called triples. Each triple has three elements - the subject, predicate and object. Each element in the triple is specified by either a Universal Resource Identifier (URI) or a literal (a string, number, etc). A collection of RDF triples can be viewed as a graph, in which the subject and object are nodes and the predicate is a directed arc that points from the subject to the object.

URI's can be used to uniquely identify information that exists on the web, in a filesystem, in a database, etc.; as well as concepts, persons, or objects that do not have an electronic representation. So if related pieces of information are stored in separate locations on a server or on the web, the relationships among them can be detailed using RDF, and a URI can be used instead of the full set of information that the URI refers to.

For example, a sensor may generate thousands of images an hour; the make and age of the sensor may help an analyst determine the quality or reliability of the images that were captured, but each image does not need to include a copy of the make/age metadata of the sensor in its pedigree. Instead, that sensor metadata can be stored in one unique location and only that location's URI will be incorporated into the pedigree metadata of each image. In addition, once an image has been transformed or fused with other information, the resulting image need not include another copy of the URI referring to that sensor metadata; instead, it will incorporate a URI to the earlier information's pedigree metadata, which in turn incorporates the sensor metadata URI. In this way, the volume of the metadata can be reduced to a linear function of the number of transformations.

\section{PMAF Architecture}

PMAF is intended for situations involving the following participants:

- One or more information object repositories. We assume that there is a unique naming system for objects obtained from each repository.

- One or more repositories of provenance metadata for the information objects. We do not assume that the provenance metadata is stored in the same repository as object information. 
- A client application that reads and writes information objects and uses PMAF to read and write the corresponding provenance metadata.

- PMAF, which provides an API for both querying and publishing provenance metadata.

The design for PMAF and how it interacts with the various participants are shown in Figure 1.

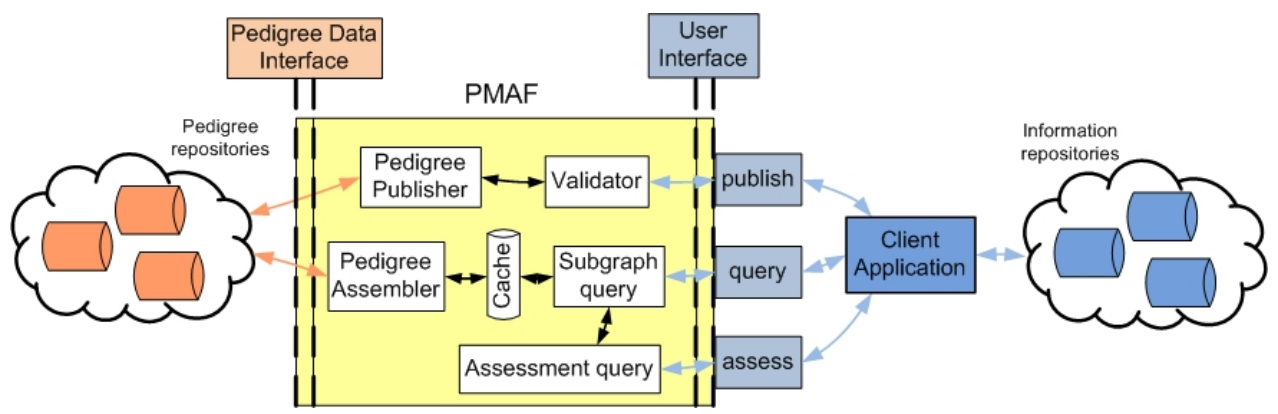

Fig. 1. PMAF Architecture

\subsection{Pedigree Assembler}

The pedigree assembler assembles the root pedigrees from pedigree fragments that it receives from one or more repositories of provenance metadata. It supports extensibility through the use of pluggable modules to handle system-specific operations that depend on the type of information repositories being used, and to use a standard framework for encoding provenance information, independent of the type of provenance storage in use.

The pedigree assembler can easily be adapted for different types of provenance storage, and for different granularities of provenance metadata. Provenance metadata can be stored in files, in a database, or as separate objects in an information object repository. Multiple sources of provenance metadata must be merged before full provenance can be assembled.

\subsection{Assessment Query}

A client application can invoke assessment queries on an object in its information repository(s) to help determine the quality and trustworthiness of the information.

\subsection{Subgraph Query}

Each assessment invokes one or more subgraph queries for the given object. The result of a subgraph query is a provenance subgraph, which is a data structure describing a particular aspect of the provenance of the object. Each request for a subgraph includes a specification for the type of subgraph desired, together with a URI that uniquely names the subject of the subgraph. The subgraph query component constructs the requested subgraph using root pedigrees that it receives from the pedigree assembler. 


\subsection{Pedigree Publisher}

A client application can insert or modify objects in its information repository(s), and make calls on PMAF to publish corresponding additions to the provenance metadata in the appropriate pedigree repository.

\subsection{Publish API}

The mapping between information object names and the corresponding provenance metadata is a modular component that can be plugged into the PMAF framework to accommodate new types of information objects. The publish API allows client applications to publish provenance metadata for new objects without needing to know the format of the metadata or where it will be stored. It makes use of the modular mapping between object names and provenance metadata.

\section{PMAF Interfaces}

PMAF includes a variety of graphical interfaces; some support pedigree browsing and drill-down, and others enable users to quickly estimate information quality based on the assessments described in the next section.

PMAF's built-in pedigree browsers provide two distinct views of the pedigree. The tree browser depicts the ancestry of a given document (or, alternatively, its descendants) in hierarchical form, where each node in the tree represents a source (or descendant) document. Such hierarchal trees are familiar to computer users from file system browsers. The graph browser depicts the pedigree as a graph, with labeled arcs to source documents, publishers, and other pedigree metadata. The graph viewer enables the user to zoom out to a bird's eye view of the whole graph or zoom in (drill down) to sections of interest.

\section{PMAF Assessments}

In time-critical situations, users need to quickly evaluate information quality. To support assessment of the quality of information objects, PMAF offers quick, accurate, and focused analyses based on the pedigree. Documents and other objects and their relationships in the pedigree that have an immediate bearing on the quality of the information are evaluated and presented to the user. Other analyses estimate the trustworthiness of information publishers or the reliability of documents.

PMAF provides the user with five assessments for an information object that help to evaluate its quality:

- $\quad$ its influence or impact on other information objects (Source Impact)

- whether it has been superseded or deprecated by newer information (Source Deprecation)

- whether it derives from conflicting sources (Conflict Notification)

- $\quad$ publisher reputation (Feedback)

- $\quad$ source corroboration analysis (Unique Sources). 
Based on the assessment analyses, the PMAF user can estimate the quality of the information. In addition, custom pedigree assessments can be created and plugged into PMAF that are specific to the information domain of a client application, provide additional dimensions of metadata analysis, etc.

\section{PMAF Vocabularies}

PMAF includes a baseline provenance ontology that extends the Dublin Core Metadata Initiative (DCMI) vocabulary, incorporating terms that support a decisionmaking process, the dependency of data in general, and the occurrence of conflicting data. In addition PMAF includes vocabulary from the Friend of a Friend (FOAF), DoD Discovery Metadata Specification (DDMS) and Intelligence Community Metadata Standard for Information Security Markings (IC-ISM) standards.

At its core PMAF is vocabulary-agnostic. PMAF does not require the use of any particular vocabulary or term, and is completely configurable to use any vocabulary whether previously defined or newly created. Rather than requiring the use of a particular provenance model or vocabulary storage format, terms are recorded using the common denominator of RDF and need not be predefined by PMAF. Depending on the use case, an integrated approach combining PMAF with another ontology tool and/or model may be beneficial.

\section{PMAF Applications}

Our recent integration efforts for PMAF have focused on document processing and content management applications. However, we believe that the open architecture of PMAF is suitable for collection of provenance metadata from a variety of system and application types, such as:

- Workflow: collect provenance for information objects as they pass through processes that run within the workflow system

- Enterprise Service Bus: report message passing and transformations

- Web Services: collect provenance for web service processing of information objects, as well as of the web services themselves (e.g. versioning)

\section{PMAF Demonstration}

We will begin the PMAF demonstration by using the graph browser interface to view the existing metadata graph for an information object, in this case a document. Since the provenance metadata is stored in RDF format, it can be viewed as a graph with the subjects and object resources represented as nodes, and each predicate as a link between a subject node and object node. The links represent metadata such as the type of the object, the creator, the publisher, the create date, sources for the object (e.g. other documents), and if the object has subparts (each of which can have its own metadata, i.e. at a fine-grained level). 
We will demonstrate interactively exploring the graph by reviewing the metadata for sources, sources of sources, etc (moving backward in time), and then understanding the impact of sources on downstream objects (moving forward in time). We will also demonstrate the results of running several of the built-in PMAF assessments on the sample provenance metadata, including the Unique Sources, Source Deprecation, Conflict Notification, and Source Impact assessments.

We will then demonstrate the integration of PMAF with a standard document editor (Microsoft Word), allowing the creation and publishing of provenance metadata as a side effect of copying and pasting information from existing files into a new document. Finally we will demonstrate the integration of PMAF with a Content Management System (Microsoft SharePoint), allowing the creation and publishing of new metadata for files based on standard CMS operations such as checking in, moving and renaming a file.

\section{References}

1. Gioioso, M.M., McCullough, S.D., Cormier, J.P., Marceau, C., Joyce, R.A.: Pedigree Management and Assessment in a Net-centric Environment. In: Proceedings of the SPIE Symposium on Defense and Security (2007)

2. Combs, V.T., Hillman, R.G., Muccio, M.T., McKeel, R.W.: Joint Battlespace Infosphere: Information Management within a C2 Enterprise. In: $10^{\text {th }}$ International Command and Control Research and Technology Symposium (2005)

3. RDF Primer, http://www.w3 . org/TR/rdf-primer

4. Berners-Lee, T.: Uniform Resource Identifiers (URI): Generic Syntax, http: //www. ietf.org/rfc/rfc2396.txt

5. Dublin Core Metadata Initiative, http: / / dublincore.org

6. Chapman, A.: Industry Provenance State of the Art, presentation to the Information Assurance Metadata Community of Practice (IAM CoP) working group (2009) 